\title{
PXR: More Than Just a Master Xenobiotic Receptor
}

\author{
Peter O. Oladimeji and Taosheng Chen \\ Department of Chemical Biology and Therapeutics, St. Jude Children's Research Hospital, Memphis, Tennessee
}

Received August 4, 2017; accepted November 3, 2017

\section{ABSTRACT}

Pregnane $X$ receptor $(\mathrm{PXR})$ is a nuclear receptor considered to be a master xenobiotic receptor that coordinately regulates the expression of genes encoding drug-metabolizing enzymes and drug transporters to essentially detoxify and eliminate xenobiotics and endotoxins from the body. In the past several years, the function of PXR in the regulation of xenobiotic metabolism has been extensively studied, and the role of PXR as a xenobiotic sensor has been well established. It is now clear, however, that PXR plays many other roles in addition to its xenobiotic-sensing function. For instance, recent studies have discovered previously unidentified roles of PXR in inflammatory response, cell proliferation, and cell migration. PXR also contributes to the dysregulation of these processes in diseases states. These recent discoveries of the role of PXR in the physiologic and pathophysiologic conditions of other cellular processes provides the possibility of novel targets for drug discovery. This review highlights areas of PXR regulation that require further clarification and summarizes the recent progress in our understanding of the nonxenobiotic functions of PXR that can be explored for relevant therapeutic applications.

\section{Introduction}

Animals are constantly exposed to environmental chemicals and toxic endogenous compounds, and to sense and protect against such, mammals have evolved a defensive network regulated by xenobiotic receptors such as pregnane $\mathrm{X}$ receptor (PXR; also known as steroid and xenobiotic receptor [SXR]; NR1I2) (Bertilsson et al., 1998; Blumberg et al., 1998; Kliewer et al., 1998), which is a well-established xenobiotic sensor. PXR induces the expression of genes encoding drugmetabolizing enzymes, such as cytochrome P450 enzymes (e.g., CYP3A4), conjugation enzymes (e.g., sulfotransferase), and transporters (e.g., multidrug resistance 1) (Rosenfeld et al., 2003; Zhou et al., 2009; Chai et al., 2013). Cytochrome P450 enzymes, conjugation enzymes, and transporters are required for the metabolism and clearance of xenobiotics in the liver and colon (Christians et al., 2005; Xie et al., 2016). Many PXR-regulated metabolizing enzymes and transporters play critical roles in xenobiotic metabolism. For instance, PXR is the main regulator of CYP3A4 (Wei et al., 2016), the most

This work was supported in part by ALSAC and the National Institutes of Health [Grant R35-GM118041 and P30-CA21765] to T.C.

http://doi.org/10.1124/mol.117.110155. abundant hepatic and intestinal phase I enzyme, which is responsible for metabolizing greater than $50 \%$ of clinically used drugs, along with many other xenobiotics and endobiotics, in humans (Harris et al., 2003). Furthermore, the expression of several of the aforementioned drug-metabolizing enzymes and transporters, in addition to that of at least 30 other genes, is under the control of the liganded PXR (Hariparsad et al., 2009). Another xenobiotic receptor, constitutive androstane receptor (CAR; also called NR1I3), also has a broad role in xenobiotic metabolism, often overlapping that of PXR (Wei et al., 2002; Handschin and Meyer, 2003). Unlike PXR, CAR, as its name suggests, is constitutively active and, thus, has relatively high basal activity as an activator of target genes even in the absence of ligand (Baes et al., 1994; Forman et al., 1998; Wei et al., 2000; Xie and Evans, 2001).

Since the initial identification of PXR in 1998 (Bertilsson et al., 1998), its canonical function as a xenobiotic receptor has been extensively studied. Its role as a xenobiotic sensor is well established, and PXR has been considered to be a master regulator of xenobiotic metabolism (Wang et al., 2012; Wallace et al., 2013; Yan and Xie, 2016). The identification of PXR as a xenobiotic sensor has proved crucial to understanding the defense mechanism of the body against toxic compounds and

ABBREVIATIONS: AF-2, activation function domain 2; ANAPC2, anaphase-promoting complex subunit 2; BaP, benzo[a]pyrene; Bcl, B-cell leukemia; CAR, constitutive androstane receptor; CDC, cell division cycle protein; Cdk, cyclin-dependent kinase; CLO, clotrimazole; Cul, cullin; DBD, DNA binding domain; DEX, dexamethasone; DR, direct repeat; ER, everted repeat; FGF, fibroblast growth factor; FOXO, class O of the Forkhead box; GADD $45 \beta$, growth arrest and the DNA damage-inducible $\beta$; HNF $4 \alpha$, hepatocyte nuclear factor $4-\alpha$; IBD, inflammatory bowel disease; IGFBP1, insulin-like growth factor-binding protein 1; LBD, ligand binding domain; MAPK, mitogen-activated protein kinase; NF-kB, nuclear factor-kB; NR, nuclear receptor; PCN, pregnenolone 16 $\alpha$-carbonitrile; PKA, protein kinase A; PPAR, peroxisome proliferator-activated receptor; PRM, post-translational modification; PTM, post-translational modifications; PXR, pregnane $X$ receptor; RBCK1, RING-B-box-coiled-coil protein interacting with protein kinase C-1; RIF, rifampicin; RXR, retinoid X receptor; SRC, steroid receptor coactivator; TLR4, Toll-like receptor 4. 
has laid the foundation for studying how xenobiotic exposure affects different diseases states (Xu et al., 2016). In recent years, however, an increasing number of new roles for PXR beyond its canonical xenobiotic-sensing function have been described. The implication of PXR in modulating hepatic glucose and energy homeostasis, thereby revealing hitherto unknown functions of PXR in obesity and insulin resistance, reveals one of a number of new, noncanonical roles for PXR (Spruiell et al., 2014a,b). PXR is also involved in atherosclerosis development and vascular functions, to mention only a few more of its activities beyond the xenobiotic-sensing function (Febbraio et al., 2000; Masuyama et al., 2000; Takeshita et al., 2001; Zhou et al., 2006b; Guy et al., 2007; Newbold et al., 2007; vom Saal and Myers, 2008; Carwile and Michels, 2011). These aforementioned functions of PXR have been reviewed in great detail (Konno et al., 2008; Wada et al., 2009; Xiao et al., 2014); thus, it will not be discussed in this review. The discoveries of hitherto unsuspected roles for PXR have ignited a new appreciation for the receptor and suggest that PXR signaling may contribute more substantially to pathophysiologic conditions than we currently understand. This recent appreciation of the wide-ranging function of PXR has prompted several laboratories to investigate other roles of the receptor. This review summarizes recent advances in the discovery of the new functions of PXR beyond its canonical role in detoxification of xenobiotics and focuses on less discussed topics, such as its involvement in inflammation, proliferation, migration, apoptosis, and DNA damage. These newly discovered functions of PXR clearly indicate that PXR is more than just a master xenobiotic receptor.

\section{PXR Regulation}

PXR is a member of the nuclear receptor (NR) superfamily, which includes the steroid, retinoid, and thyroid hormone receptors. Members of the NR superfamily function as ligandregulated transcription factors and play critical roles in nearly every aspect of development and adult physiology (Mangelsdorf et al., 1995). PXR shares a common domain structure with other members of the NR superfamily that includes a highly conserved DNA-binding domain (DBD) with two zinc fingers (Carnahan and Redinbo, 2005; Wallace et al., 2013). By means of this DBD, PXR targets short stretches of DNA, termed response elements, in the regulatory regions of target genes. The carboxy-terminal region of PXR includes the conserved ligand-binding domain (LBD). The LBD serves as the docking site for ligands and also contains dimerization motifs and transcriptional activation domains, such as activation function 2 (AF-2). The binding of ligand to the LBD results in a conformational change in the AF-2 helix, and this change allows PXR to interact with accessory proteins and regulate the expression of target genes (Mangelsdorf et al., 1995; Bourguet et al., 2000). Because of its function as a xenobiotic sensor, PXR has evolved to recognize and accommodate a wide array of structurally different ligands, and this is possible because of its large and flexible ligand-binding pocket (Watkins et al., 2001). An important mechanistic aspect of NR engagement is that the response of an NR to a particular ligand in a given tissue is determined by the set of regulatory partners such as DNA and proteins with which that NR interacts after undergoing ligand-induced alterations such as structural changes or post-translational modifications
(PTMs) that generate, expose, or remove surfaces for interactions (McKenna et al., 1999; Gronemeyer et al., 2004; Ahmad et al., 2013). Therefore, the action of PXR, like that of other NRs, involves a number of proteins, ranging from other NRs and transcriptional coactivators to transcriptional corepressors (Gronemeyer et al., 2004).

Genetic and/or biochemical data have revealed a variety of factors-generally components of multiprotein transcriptional complexes - that mediate NR functions, including those of PXRs (McKenna et al., 1999; Glass and Rosenfeld, 2000). When bound to and activated by ligand, PXR activates target gene transcription as a heterodimer with retinoid $\mathrm{X}$ receptor (RXR) (Goodwin et al., 1999). The DBD of PXR facilitates DNA binding specificity by two highly conserved zinc-finger motifs, as well as a P-box motif and a D-box motif that allow the receptor to target and bind its xenobiotic response enhancer modules located in the $5^{\prime}$ promoter region of PXR target genes (Umesono and Evans, 1989). PXR can bind to a variety of DNA response elements, including the direct repeats (DRs) DR-3, DR-4, and DR-5 and the everted repeats (ERs) ER-6 and ER-8 (Orans et al., 2005). In a genome-wide study of PXR-regulated targets in mouse liver, Cui et al. (2010) uncovered a previously unknown PXR DNA-binding motif of DR- $(5 n+4)$ for which the receptor has a high preference. Many transcriptional cofactors that regulate PXR activity with respect to target gene promoters have been identified. These coregulators include members of the p160 family, such as steroid receptor coactivator (SRC) 1, transcriptional mediators/intermediary factor 2 (also known as SRC-2), and amplified in breast cancer-1 (also known as SRC-3), as well as suppressor for gal 1, receptorinteracting protein 140 , peroxisome proliferator-activated receptor (PPAR)- $\alpha$-binding protein, and PPAR $\gamma$ coactivator 1 (Timsit and Negishi, 2007). Signaling cascades have also been implicated in modulating PXR activity, one example being the potentiation of PXR-mediated CYP3A induction by the protein kinase A (PKA) activator forskolin, which results from enhanced recruitment of PPAR y coactivator 1 (Ding and Staudinger, 2005a). Moreover, PXR is phosphorylated by PKA in vitro, and specific activation of PKA by 8-bromo-cAMPenhanced interactions of PPAR-binding protein and SRC-1 with PXR. In contrast, protein kinase $\mathrm{C}$ represses PXR signaling (Ding and Staudinger, 2005b).

The subcellular localization of PXR in its transcriptionally inactive state remains a point of contention. Different research groups have provided conflicting evidence to support their respective positions. Squires et al. (2004) showed that inactive PXR is predominantly sequestered in the cytoplasm of mouse liver by the cytoplasmic CAR retention protein-heat shock protein 90 complex. When bound to and activated by ligand, PXR dissociates and translocates to the nucleus, where it heterodimerizes with RXR and activates target gene transcription (Squires et al., 2004). The functional motifs, namely the nuclear localization sequence (localized in the C-terminal region of the DBD), and the transcription AF-2 domain, were found to be necessary for pregnenolone $16 \alpha$ carbonitrile (PCN; mouse PXR agonist)-induced PXR nuclear translocation. It was also found that downregulating cytoplasmic CAR retention protein by means of small interfering RNA attenuated ligand-induced PXR transcriptional activation, providing additional evidence of the importance of cytoplasmic sequestration in regulating PXR transcriptional activity (Squires et al., 2004). Consistent with this observation, a 
more recent study by van de Winkel et al. (2011) showed that PXR, upon stimulation with lithocholic acid (bile acid), translocates to the nuclei in esophageal adenocarcinoma cells. In contrast to these observations, other groups have reported that human PXR is consistently localized to the nucleus, regardless of ligand binding or its activation status. Kawana et al. (2003) used transient expression in HeLa cells to show that PXR was localized to the nucleus in the absence of ligand. The same group also identified a nuclear localization signal in the DBD of PXR. Removal of the DBD resulted in solely cytoplasmic localization, and mutation of the putative nuclear localization signal resulted in PXR redistribution to both the cytoplasm and the nucleus (Kawana et al., 2003). Consistent with those findings, immunostaining assays revealed human PXR to be located solely in the nucleus both in the presence and absence of ligand (Koyano et al., 2004). The inconsistencies between these various reports may be due to the species of PXR studied (human versus mouse) or the models used in the studies (in vitro versus in vivo). The findings also suggest that protein trafficking may be important in regulating PXR activity. Nevertheless, regardless of the merits of either side in this debate, nuclear localization is clearly essential for the activation of PXR transcriptional activity.

The class I nuclear (steroid hormone) receptors, such as the estrogen and androgen receptors, function as homodimers, whereas class II NRs, such as PXR, act as heterodimers with $\mathrm{RXR} \alpha$ (Kliewer et al., 1998). In an attempt to understand the structural basis of how PXR interacts with xenobiotics, Watkins et al. (2003) solved the crystal structure of the human PXR LBD in the presence of a peptide of human SRC-1 (residues 676-700) and the cholesterol-lowering compound SR12813. Interestingly, they also found that PXR LBD molecules form a homodimer and that the homodimer interface is formed primarily between the $\beta 1^{\prime}$ strands of each monomer, different from the interacting surface for its obligate RXR binding partner. Furthermore the terminal $\beta$-strands in each of these $\beta$-sheets associate in an antiparallel fashion to generate the PXR homodimer, which produces a 10 -strand intermolecular antiparallel $\beta$-sheet. This form of homodimerization is unique to PXR. The size of the homodimer interface is large, suggesting that it is of functional relevance. Noble et al. (2006) later confirmed the discovery by Watkins et al. (2003) that PXR LBD forms homodimers in solution using a sedimentation experiment. The mutation of tryptophan 223 and 225 both to alanine (Trp223Ala and Tyr225Ala) on the PXR homodimer interface prevents the formation of the PXR homodimer, significantly reduces the ability of PXR to respond to ligands, and results in PXR exhibiting no basal transcriptional activation (Noble et al., 2006). This observation indicates that mutations that eliminate PXR homodimer formation substantially reduce the ability of the receptor to upregulate gene expression in a ligand-dependent or ligand-independent fashion (Noble et al., 2006). The PXR mutant containing Trp223Ala and Tyr225Ala was able to form a complex with RXR; however, the recruitment of coactivators such as SRC-1 was impaired, suggesting that the PXR homodimer is integral to cofactor recruitment and hence to PXR transcriptional activity (Noble et al., 2006).

Although it is known that the transcriptional activity of PXR is governed by direct binding of ligands, many reports have indicated that cellular signaling pathways modulate the functions of NRs, including PXR. NR function is regulated by PTMs such as phosphorylation, acetylation, sumoylation, methylation, myristoylation, nitration, ADP-ribosylation, and/or isoprenylation. Several investigations have yielded direct evidence that these NR PTMs are responsible for the progression of many diseases, including cancers, diabetes, and obesity (Anbalagan et al., 2012). Additionally, PTMs of NRs provide an important mechanism for cross talk between signaling pathways, thus underscoring the involvement of PXR in several physiologic processes and suggesting that there are nonliganded mechanisms of PXR regulation, and an instance of this was reported by Biswas et al. (2011). PTMs, such as phosphorylation of PXR through PKA signaling, have been shown to modulate PXR activity and the recruitment of cofactors such as SRC-1 and NR corepressor. This regulation of PXR by PKA was shown to modulate the involvement of PXR in the inflammatory response (Lichti-Kaiser et al., 2009). Cyclin-dependent kinase (Cdk) 2, a kinase that is important in regulating cell-cycle progression, was shown to attenuate PXR transcriptional activity during cell-cycle progression (Lin et al., 2008). Along the same lines, Sugatani et al. (2010) showed that Cdk2 negatively regulated xenobiotic metabolism genes. PXR stabilization through ubiquitination has also been described. The E3 ubiquitin ligase RING-B-box-coiled-coil protein interacting with protein kinase C-1 (RBCK1) is a direct binding partner of PXR, and RBCK1 ubiquitinates PXR, resulting in PXR degradation (Rana et al., 2013). Furthermore, suppressor for gal 1, a subunit of the $26 \mathrm{~S}$ proteasome, interacts with PXR in the presence of specific ligands, suggesting that different PXR ligands elicit varying characteristics on PXR such as accumulation in cells and, potentially, on PXR functions in cells (Masuyama et al., 2002). Several groups have demonstrated the involvement of acetylation in PXR function regulation (Biswas et al., 2011; Sugatani et al., 2012); however, the histone acetyltransferase involved in this process remains to be determined. It is possible that the p160 coactivators recruit the histone acetyltransferase involved in the acetylation of PXR to the PXR transcriptional machinery.

Despite the discovery of the mode of regulation of PXR and the acquisition of knowledge regarding its localization, several questions remain (Fig. 1, A-C). 1) Because the interacting surfaces of the PXR-PXR homodimer and PXR-RXR heterodimer are different, and the mutation that inhibited PXR-PXR dimerization did not affect PXR-RXR dimer formation but decreased PXR transcriptional activity, does PXR function in a trimer consisting of two PXR molecules and one RXR molecule (Fig. 1C)? 2) Does the dimerization dynamic (i.e., PXR-RXR heterodimer versus PXR-PXR homodimer versus PXR-PXR-RXR trimer) influence the target gene regulated by PXR (Fig. 1, A-C)? 3) Does the subcellular localization of PXR dictate the other noncanonical functions of PXR? 4) Does the specific ligand that binds PXR or the factors that interact with PXR dictate its specific role? 5) And how does the DBD of PXR affect the function of PXR, and does DNA-bound PXR behave differently from the non-DNA-bound form? These are questions that require further clarification. It is beyond the scope of this article to connect all the nonxenobiotic roles of PXR with the mode of regulation; however, we will discuss recent discoveries concerning the functions of PXR beyond its canonical xenobiotic sensor role. 


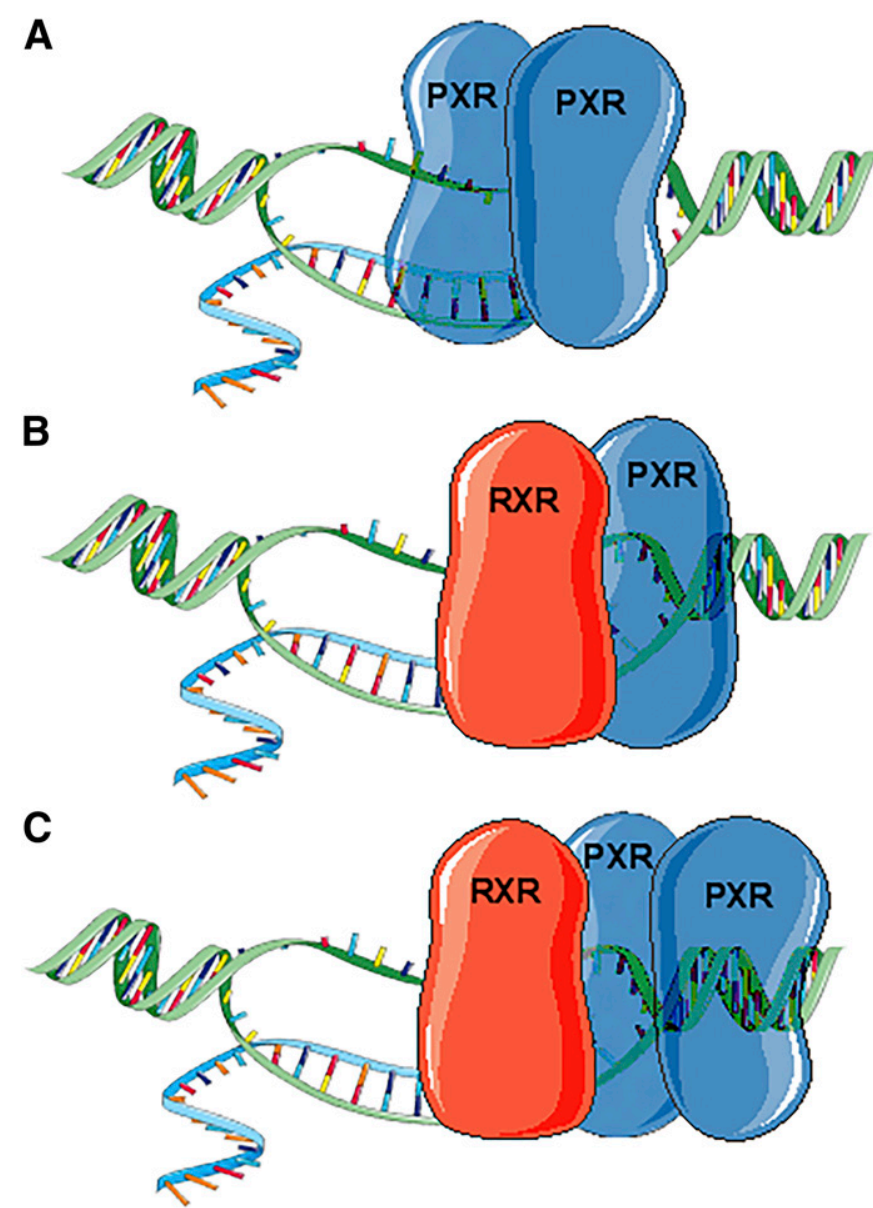

Fig. 1. Schematic representation of the possible PXR dimers and trimer. (A) PXR forms a homodimer and binds to PXR response elements within the promoter of the target gene. Mutation to the PXR-PXR interacting surface has been shown to decrease PXR transcriptional activity. (B) PXR forms a heterodimer with RXR, its obligate binding partner, to regulate the transcription of target genes. (C) PXR can potentially form a PXR-PXR-RXR trimer because the interacting surfaces of PXR homodimer and PXR-RXR heterodimer are different. PXR-RXR dimer formation in regulating PXR activity is well established. PXR-PXR dimer formation is relatively new and requires further exploration to gain a better understanding of its function. The idea that PXR-PXR-RXR trimer can potentially form in cells and may be required for full PXR transcriptional activity is thought provoking and warrants investigation.

\section{PXR in Inflammation}

The integrity of the gut lining is important for preventing intestinal injury and maintaining the gut barrier function. It is now clear that microbes, food, and metabolites regulate the gut barrier function through immune recognition (Turner, 2009). Several bowel diseases, such as inflammatory bowel disease (IBD), intestinal ischemia, graft-versus-host disease, Crohn's disease, ulcerative colitis, and celiac disease, are associated with dysfunction and chronic inflammation of the intestinal tract (Tamion et al., 1997; Baert et al., 1999; Brown et al., 1999; Suenaert et al., 2002; Duerksen et al., 2005; Heller et al., 2005; Blair et al., 2006; Zeissig et al., 2007). Gene expression profiling of inflamed colon tissues from patients with ulcerative colitis or Crohn's disease identified several downregulated detoxification genes and ATP-binding cassette transporters. Along with the decrease in the expression of several phase II enzymes and xenobiotic transporters, there was a significant downregulation of PXR, suggesting that PXR plays a role in the pathogenesis of IBD (Langmann et al., 2004). In addition, several single nucleotide polymorphisms linked to decreased PXR activity or expression have been identified in patients with IBD (Dring et al., 2006). PXR was shown to play a prominent role in maintaining the intestinal wall integrity by regulating inflammation in hepatocytes and the small intestine. PXR activation inhibited the action of nuclear factor $-\kappa \mathrm{B}(\mathrm{NF}-\kappa \mathrm{B})$ on the expression of its target genes. This PXR-dependent inhibition of NF- $\kappa \mathrm{B}$ was potentiated by PXR agonists (Zhou et al., 2006a). In a mouse model in which acute IBD was induced by administering $2.5 \%$ dextran sulfate sodium in the drinking water, mice treated with the PXR agonist PCN were protected from dextran sulfate sodium-induced colitis when compared with vehicle-treated mice. Indole 3-propionic acid, an indole metabolite produced exclusively by gut microflora (Wikoff et al., 2009), was identified as a possible physiologic ligand for PXR. In mouse intestine, indole 3-propionic acid downregulated enterocytemediated inflammatory cytokine tumor necrosis factor- $\alpha$ and upregulated junctional protein markers. Furthermore, intestinal epithelial cells from $\mathrm{Nr} 1 i^{-1-}$ mice were shorter, more loosely packed, and more permeable than those of the corresponding cells of $\mathrm{Nr}_{1 i 2^{+/+}}$mice. $\mathrm{Nr} \mathrm{i2}^{-/-}$mouse intestinal cells exhibited upregulation of Toll-like receptor 4 (TLR4), and the overall defects in the epithelial barrier were corrected in $\mathrm{Nr} 1 \mathrm{i}^{-1-} \mathrm{Tlr} 4^{-{ }^{-}-}$mice (Venkatesh et al., 2014), suggesting that PXR plays an anti-inflammatory role by negatively regulating TLR4.

\section{PXR in Cell Proliferation}

The PXR activators dexamethasone (DEX) and PCN have long been known to induce mitogenesis in the liver resulting in hepatomegaly (Thatcher and Caldwell, 1994). More recently, Shizu et al. (2013) found that the stimulation of PXR alone with PCN was insufficient to induce cell proliferation in mouse liver, but PCN bolstered CAR-mediated hepatocyte proliferation induced by the CAR agonists 1,4-bis[2-(3,5dichloropyridyloxy)]benzene and phenobarbital, as well as PPAR $\alpha$-mediated hepatocyte proliferation induced by Wy-14643 (a PPAR $\alpha$ ligand). The enhancing effects of PCN cotreatment were not observed in PXR-deficient mice, suggesting that PXR plays a unique role in the cell cycle of murine hepatocytes, enhancing CAR- and PPAR $\alpha$-mediated hepatocyte proliferation without itself inducing proliferation (Shizu et al., 2013). In the same study, the authors demonstrated that PCN treatment increased the RNA content of quiescent cells and decreased hepatic levels of mRNAs encoding p27 and p130, both of which negatively regulate the re-entry of quiescent cells into the cell cycle (Shizu et al., 2013). Overexpression of p130 in the HepG2 liver hepatocellular carcinoma cell line led to growth suppression, G0 cell-cycle arrest, and a reduction in tumorigenicity in SCID mice (Huynh, 2004). Additionally, after initiation with diethylnitrosamine, phenobarbital significantly promoted liver tumorigenesis in p27-deficient mice, compared with wild-type mice (Sun et al., 2008), suggesting that PXR modulates inhibitors of cell-cycle progression. Members of class $\mathrm{O}$ of the Forkhead box (FOXO) transcription factors control the G0/G1 and G1/S phase progressions of cells, and FOXO-mediated regulation of the cell cycle depends on the transcriptional upregulation of the cell-cycle inhibitor genes (Eijkelenboom and Burgering, 2013). 
FOXO3 overexpression abrogated the enhancing effect of PXR on serum-mediated proliferation (Shizu et al., 2016). Together, these observations suggest that activated PXR prevents FOXO3-mediated transcription of cell-cycle suppressor genes, because G0/G1 and G1/S checkpoints are the ratelimiting stages during cell-cycle progression. This might enable hepatocytes to pass these checkpoints more easily and accelerate cell-cycle progression, making the genetic information of the cells error prone and potentially resulting in cancerous cells. These findings also explain the observed hepatocyte proliferation induced by growth factors and cytokines during liver regeneration.

Other studies have shown that PXR upregulated the p21 protein, a Cdk inhibitor, to suppress the proliferation of colon cancer cells and that ectopic expression of PXR in neuroblastoma cells resulted in growth suppression (Misawa et al., 2005; Ouyang et al., 2010). Another study showed that PXR activation inhibited T-lymphocyte proliferation and compromised T-lymphocyte function (Dubrac et al., 2010). Furthermore, rifampicin (RIF)-induced activation of PXR inhibited the proliferation of cervical cancer cells, and, in a xenograft model, cells overexpressing PXR formed smaller tumors than did cells in the vector control group (Niu et al., 2014). This growth inhibition occurred as a result of G2/M phase arrest in the cell cycle. In PXR-mediated G2/M arrest, the expression of cullin (Cul) 1, Cul2, Cul3, and mitotic arrest deficient 2-like 1, which are positively correlated with cell-cycle arrest in G2/M phase, were low in control cells but high in the PXRoverexpressing cells. Additionally, anaphase-promoting complex subunit 2 (ANAPC2) and cell division cycle protein (CDC) 20 or CDC25, which are also related to the G2/M phase progression, were downregulated by PXR (Niu et al., 2014). Zhuang et al. (2011) observed a similar inhibition of proliferation in liver hepatocarcinoma cells stimulated with RIF. Flow cytometry analysis of RIF-treated cells revealed an arrest of cells in the G0/G1 phase in a PXR-dependent manner (Zhuang et al., 2011). Based on evidence that has been presented, it appears that PXR may play a dual role both as an accelerator or a brake of cell proliferation. When acting as an enhancer, PXR predominantly exerts its effects in the G0/ G1 or G1/S phases where its activation suppresses cell cycle suppressor genes like p27 and p130 (Fig. 2). On the other hand, when acting as an inhibitor of proliferation, its effects are primarily observed in the G2/M phase of the cell cycle where p21 is enhanced but a cell cycle promoter like CDC20 and CDC25 are suppressed (Fig. 2). It is still unclear whether cellular or tissue context plays a role in dictating whether PXR acts as an enhancer or an inhibitor of proliferation. Additionally, the other factors that may push the PXR proliferative function in one direction as opposed to the other are yet unknown.

Many drugs act on certain phases of the cell cycle to induce cell death. For example, mercaptopurine, methotrexate, and cytarabine act on the $\mathrm{S}$ phase by interfering with DNA synthesis, whereas vincristine and vinblastine act on the M phase to induce cell death (Binet et al., 1990). PXR is a xenobiotic receptor that regulates drug metabolism, and overactivation of this mechanism may result in drug resistance. However, the observations described here suggest that proliferation inhibition by PXR activation might be an important mechanism in desensitization to drugs and the maintenance of cell viability. That is, PXR may act to keep cells in a
G2 / M phase

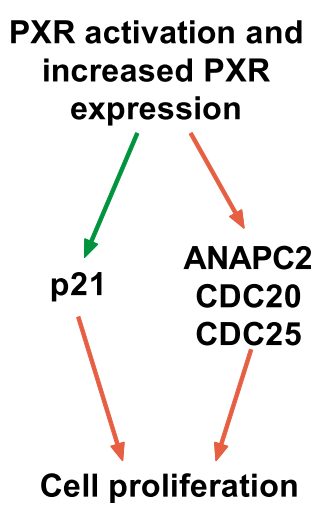

G0-G1/S phase

PXR activation

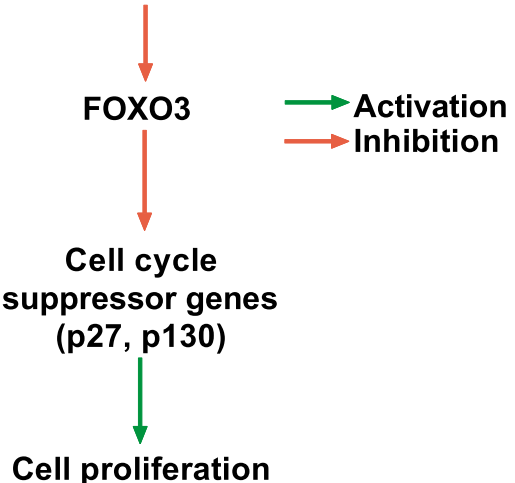

Cell proliferation
Fig. 2. PXR serves a dual role in regulating cell proliferation. PXR could inhibit cell proliferation by inhibiting the G2-M phase progression of cell cycle (left panel), or enhance cell proliferation by imposing its effects in the G1-S phase transition of cell cycle. The driving factor for what role to favor remains unknown, but whatever role is favored is predominant in that model.

state or cell-cycle phase in which they are not sensitive to drugs in their milieu, further emphasizing the broader roles of PXR.

\section{PXR in Cell Motility}

Cell migration is a widespread and complex process that is crucial to the morphogenesis of animal body plans and individual organ systems. Additionally, the activation of cell migration underlies the invasion and metastasis of human cancers, making the study and understanding of cell motility clinically relevant. Kodama and Negishi (2011) showed that PXR can induce motility in hepatocellular carcinoma cells. They observed that PXR activation by RIF stimulated the phosphorylation of p38 mitogen-activated protein kinase (MAPK). Subsequent microarray analysis revealed that the gene encoding growth arrest and the DNA damage-inducible $\beta$ (GADD $45 \beta$ ) was induced before activation of the p38 MAPK signal pathway after RIF treatment (Fig. 3) (Kodama and Negishi, 2011). Furthermore, PXR activation resulted in a morphologic change in cells, reorganization of actin filaments, and enhanced cell migration (Kodama and Negishi, 2011). The same group subsequently discovered an additional signaling pathway that regulated cell migration, in which RIF-induced activation of PXR suppressed hepatocyte nuclear factor 4- $\alpha$ $(H N F 4 \alpha)$ gene, resulting in the upregulation of insulin-like growth factor-binding protein (IGFBP) genes (Fig. 3) (Kodama et al., 2015). In another study, Wang et al. (2011) showed that PXR activation in response to RIF increased the migration potential of colon cancer cells and metastasis to the liver of cells injected into the spleen. The PXR-dependent tumor invasiveness was found to be dependent on fibroblast growth factor (FGF) 19, and FGF 19 is a direct target gene of PXR (Fig. 3) (Wang et al., 2011).

Kodoma and colleagues (Kodama and Negishi, 2011; Kodama et al., 2015) demonstrated PXR GADD45 $\beta$-p38 MAPK and PXR-HNF4 $\alpha$-insulin-like growth factor-binding protein 1 (IGFBP1) signaling axes, both of which regulate cell 


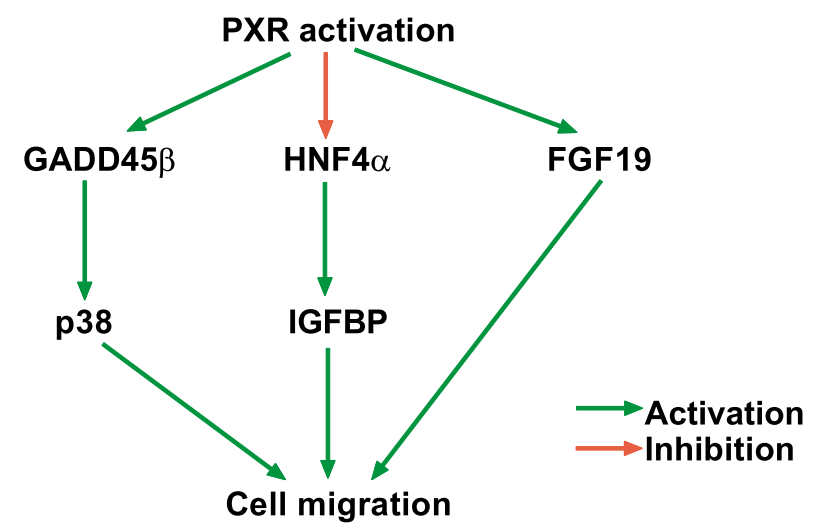

Fig. 3. PXR enhances cell migration through the GADD $45 \beta$-p38, HNF $4 \alpha$ IGFBP1, and FGF19 regulatory axes.

morphology and cell motility. The PXR-dependent regulation of cell migration via the genes encoding GADD $45 \beta$ and IGFBP1 underscores the possibility that PXR plays diverse roles in cell regulation, because both GADD $45 \beta$ and IGFBP1 regulate various cellular processes, including apoptosis, the cell cycle, DNA repair, cell proliferation, and cell migration, through the interactions of GADD $45 \beta$ with various signal factors, such as the cdc2/cyclin B1 complex, p38, p21, c-Jun $\mathrm{N}$-terminal kinase, and proliferating cell nuclear antigen (Liebermann and Hoffman, 2008; Salvador et al., 2013), and through the interaction of IGFBP1 with IGF (Jones and Clemmons, 1995; Chesik et al., 2010).

Wang et al. (2011) connected PXR to the malignant phenotype of cancer cells. Previous studies have demonstrated that PXR, which is expressed in various cancers, such as colon, ovary, breast, endometrial, and prostate cancers, contributes to tumor progression and drug resistance by inducing enzymes such as CYP3A4, thereby enabling the metabolism of therapeutic agents and steroid hormones such as estrogens (Chen and Nie, 2009; Qiao et al., 2013). Taken together, these findings suggest that PXR drives both of the processes that account for poor prognosis in cancer-metastasis and drug resistance. Because several environmental xenobiotics and food components can activate PXR at clinically relevant concentrations in humans (Harris et al., 2003; Nowack et al., 2009), the implications that the environment and diet exert an effect on cancer progression through PXR are radically thought provoking and warrant careful consideration.

\section{PXR in Apoptosis}

Apoptosis is an important mechanism by which organisms minimize the toxic effects of xenobiotics. It is a form of innate cell suicide executed by caspases and is critical for cell turnover and, hence, for the maintenance of tissue homeostasis. Apoptosis can be triggered by a variety of external and inherent signals; it occurs spontaneously in untreated malignant neoplasms and is involved in at least some types of therapeutically induced tumor regression in the form of synthetic lethality. Apoptosis has also been implicated in the physiologic shrinkage and atrophy of various tissues and organs (Kerr et al., 1972; Fadeel and Orrenius, 2005). The ability of cells to escape apoptosis is a hallmark of cancer cells.
In the past decade, PXR has been implicated in this ubiquitous but important physiologic process (Bailly-Maitre et al., 2001; Zucchini et al., 2005; Masuyama et al., 2007; Zhou et al., 2008). Bailly-Maitre et al. (2001) observed that DEX, a prototypical CYP3A inducer and PXR activator, could inhibit spontaneous apoptosis in hepatocyte primary cultures by upregulating the antiapoptotic proteins B-cell leukemia (Bcl) 2 in human hepatocytes and Bcl-xL in rat hepatocytes, whereas the expression of the proapoptotic proteins Bcl-xS and Bcl-2-associated death promoter was not detected or remained unchanged. Building on this study, Zucchini et al. (2005) showed that seven known human and/or rat PXR activators (DEX, also a glucocorticoid receptor ligand (Weikum et al., 2017), RIF, phenobarbital, clotrimazole (CLO), spironolactone, PCN, and metyrapone] protected cells against staurosporine-induced apoptosis by upregulating Bcl-xL and Bcl-2 in rat and human hepatocytes. They observed that the knockdown of PXR expression by antisense technology in rat hepatocytes inhibited the Bcl-xL upregulation induced by CLO and that the overexpression of PXR in HepG2 cells increased Bcl-2 expression upon CLO treatment and protected the cells against Fas-induced apoptosis (Zucchini et al., 2005). This suggests that PXR plays a role in promoting hepatocyte survival by upregulating the $\mathrm{Bcl}-\mathrm{xL}$ and Bcl-2 anti-apoptotic proteins. In a later study, Zhou et al. (2008) further confirmed that PXR had an antiapoptotic role. They showed that the expression of constitutively activated PXR or the pharmacologic activation of PXR by the agonist RIF in PXR-overexpressing cells protected HCT116 human colon carcinoma cells from deoxycholic acid-induced apoptosis. Interestingly, PXR activation also protected HCT116 cells from adriamycin-induced cell death, suggesting that the antiapoptotic effect of PXR is not specific to a particular compound but may protect cells from a variety of apoptosis inducers (Zhou et al., 2008).

Pharmacologic activation of endogenous PXR in LS180 colorectal adenocarcinoma cells also inhibited staurosporineinduced apoptosis. Mechanistically, the antiapoptotic effect of PXR appeared to be independent of its xenobiotic function, because HCT116 cells exhibited little basal or inducible expression of bile acid-detoxifying enzymes that are transcriptional targets of PXR. SuperArray analysis showed that PXR-mediated inhibition of deoxycholic acid-induced apoptosis was associated with the upregulation of multiple antiapoptotic genes, including BAG3 (Bcl-2-associated athanogene 3) and the genes encoding BIRC2 (baculoviral apoptosis inhibitory protein repeat-containing protein 2) and MCL-1 (myeloid cell leukemia 1), and downregulation of proapoptotic genes, such as BAK1 (Bcl-2 antagonist/killer 1) and TP53/p53 (Zhou et al., 2008). The antiapoptotic effect was also observed in constitutively activated PXR transgenic mice treated with lithocholic acid, a known apoptotic bile acid in vivo. Furthermore, PXR transgenic mice were sensitized to dimethylhydrazineinduced colonic carcinogenesis, supporting the idea that PXR participates in the malignant transformation of cells (Zhou et al., 2008).

Recently, Robbins et al. (2016) proposed an intriguing model of the antiapoptotic effect of PXR. They showed that PXR bound and sequestered the tumor suppressor p53, thus decreasing the binding of p53 to its target gene promoters. The PXR-p53 interaction sequesters p53, thereby inhibiting p53 transcriptional activity, enhancing malignant 
transformation, and protecting cells from drug-induced apoptosis (Elias et al., 2013; Robbins et al., 2016). This recent model of a physical and mutually inhibitory interaction between PXR and p53 provides further mechanistic insight into the previously observed regulation of proapoptotic and antiapoptotic genes that are targets of p53 in response to PXR activators.

\section{PXR in DNA Damage}

The DNA of eukaryotic cells is under constant bombardment from chemicals, free radicals, and/or ionizing radiation as a result of environmental exposure, the byproducts of intracellular metabolism, or medical therapy. DNA damage repair proteins sense the damage brought on by this constant attack and initiate the recruitment of protein complexes to the site of the genotoxic lesion (reviewed in Hakem, 2008). DNA damage in terminally differentiated cells (e.g., muscle cells) prompts DNA damage repair to ensure the integrity of the transcribed genome, but the induction of DNA damage in dividing cells results in the activation of the cell cycle G1/S, intra-S, and G2/M checkpoints. These checkpoints halt the cell-cycle progression to enable the DNA damage repair machinery to do its work, thereby avoiding the transmission of incorrect genetic information to the progeny. The DNA damage response during any phase of the cell cycle follows the same crucial steps. When DNA damage is detected by sensor proteins, signal transducer proteins transduce the signal to effector proteins that launch a cascade of events that leads to cell-cycle arrest, apoptosis, DNA repair, and/or the activation of damage-induced transcription programs (Houtgraaf et al., 2006). It is clear that the downregulation of DNA damage surveillance and repair mechanisms plays a critical role in tumor progression and increases the genetic and epigenetic instability required for the uncontrolled proliferation and adaptability associated with aggressive tumors.
In a study by Naspinski et al. (2008), liver carcinoma cells were exposed to benzo[a]pyrene (BaP), a common environmental contaminant that is found in air, water, soil, sediment, and cooked foods, and that is also a well-established animal carcinogen and a probable human carcinogen. These authors found that PXR protected cells from BaP-induced DNA damage by upregulating metabolizing enzymes that contribute to the detoxification of the compound (Naspinski et al., 2008). A later study showed that ginsenoside, the main active ingredient in Panax ginseng and a functional ligand of PXR, protected human dermal fibroblasts from BaP-induced DNA damage. A mechanistic study revealed that ginsenoside increased the expression of the gene encoding NADPH:quinine oxidoreductase 1, an important phase II detoxifying enzyme, by activating the phosphatidylinositol 3-kinase/Akt/Nrf2 pathway. The involvement of PXR in the cytoprotective effect of ginsenoside against BaP-induced DNA damage was confirmed by subsequent knockdown of PXR with small interfering RNA (Poon et al., 2012).

\section{Conclusion}

We have just begun to recognize the importance of the nonxenobiotic functions of PXR and the impact of factors that redirect PXR signaling. In each of these cases, molecular biology has provided us with a variety of concepts as to how PXR and/or its ligands can induce or modulate important physiologic programs (Fig. 4). The challenge now is to generate chemical probes that selectively interrogate one or some of these functions to achieve the pharmacologically desired effects. Can specific nodes in the numerous PXR signaling pathways be identified to specifically target a process without affecting others? Undoubtedly, elucidating the other roles of PXR in physiologic or pathophysiologic processes represents both a major challenge for PXR pharmacology and a potential opportunity to identify new targets for drug discovery.

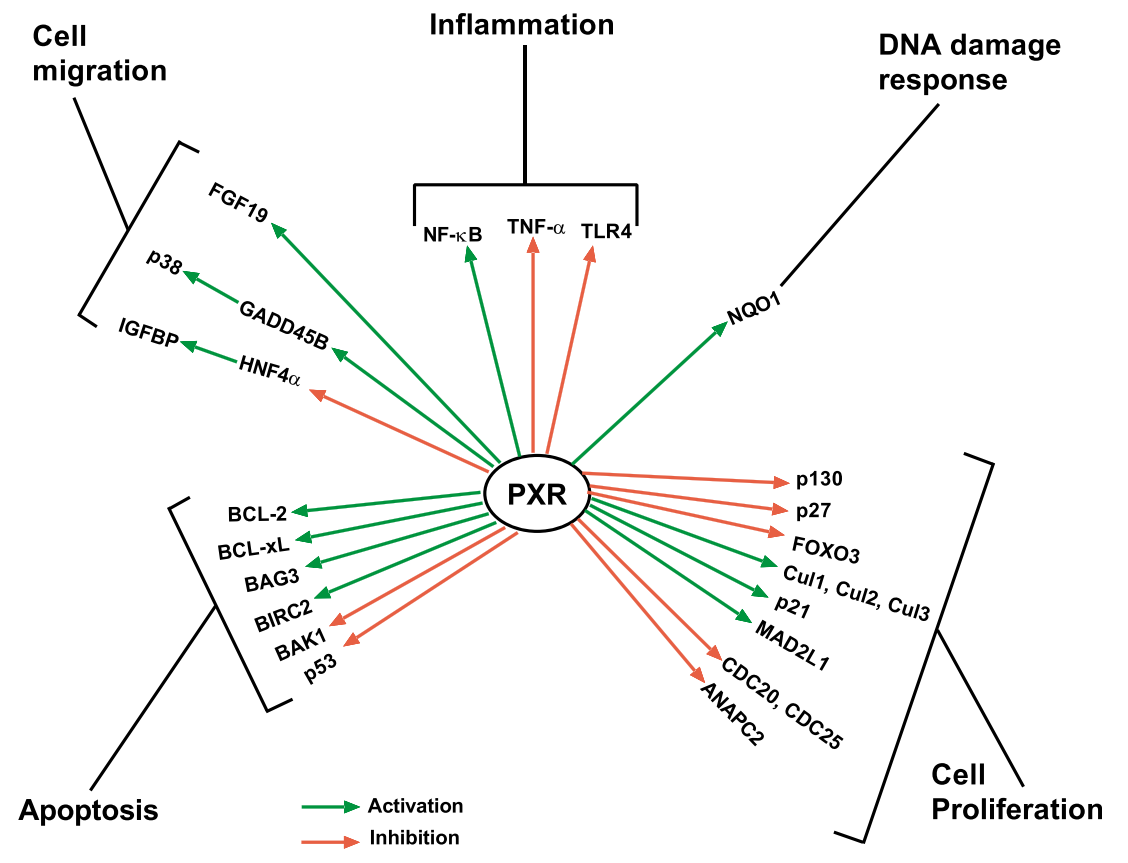

Fig. 4. Schematic summary of nonxenobiotic functions of PXR. It has become clearer that PXR plays critical roles in physiologic and pathophysiologic states beyond its canonical xenobiotic-sensing function. BAK1, Bcl-2 antagonist/killer 1; BIRC2, baculoviral apoptosis inhibitory protein repeat-containing protein 2; MAD1, mitotic arrest-deficient 1 ; TNF- $\alpha$, tumor necrosis factor$\alpha$. 


\section{Acknowledgments}

The authors thank Dr. Keith A. Laycock, Senior Scientific Editor of the Scientific Editing Department of St. Jude Children's Research Hospital, for comprehensively editing this manuscript. Images used to create Figure 1 are adapted from nucleic acids and intracellular components by SMART Servier Medical Art, used under agreement CC BY 3.0.

\section{Authorship Contributions}

Wrote or contributed to the writing of the manuscript: Oladimeji, Chen.

\section{References}

Ahmad E, Rabbani G, Zaidi N, Khan MA, Qadeer A, Ishtikhar M, Singh S, and Khan $\mathrm{RH}$ (2013) Revisiting ligand-induced conformational changes in proteins: essence, advancements, implications and future challenges. J Biomol Struct Dyn 31: $630-648$.

Anbalagan M, Huderson B, Murphy L, and Rowan BG (2012) Post-translational modifications of nuclear receptors and human disease. Nucl Recept Signal 10:e001. Baert FJ, D'Haens GR, Peeters M, Hiele MI, Schaible TF, Shealy D, Geboes K, and Rutgeerts PJ (1999) Tumor necrosis factor alpha antibody (infliximab) therapy profoundly down-regulates the inflammation in Crohn's ileocolitis. Gastroenterol ogy 116:22-28

Baes M, Gulick T, Choi HS, Martinoli MG, Simha D, and Moore DD (1994) A new orphan member of the nuclear hormone receptor superfamily that interacts with a subset of retinoic acid response elements. Mol Cell Biol 14:1544-1552.

Bailly-Maitre B, de Sousa G, Boulukos K, Gugenheim J, and Rahmani R (2001) Dexamethasone inhibits spontaneous apoptosis in primary cultures of human and rat hepatocytes via Bcl-2 and Bcl-xL induction. Cell Death Differ 8:279-288.

Bertilsson G, Heidrich J, Svensson K, Asman M, Jendeberg L, Sydow-Bäckman M, Ohlsson R, Postlind H, Blomquist P, and Berkenstam A (1998) Identification of a human nuclear receptor defines a new signaling pathway for CYP3A induction. Proc Natl Acad Sci USA 95:12208-12213.

Binet S, Chaineau E, Fellous A, Lataste H, Krikorian A, Couzinier JP, and Meininger $\mathrm{V}$ (1990) Immunofluorescence study of the action of navelbine, vincristine and vinblastine on mitotic and axonal microtubules. Int J Cancer 46:262-266.

Biswas A, Pasquel D, Tyagi RK, and Mani S (2011) Acetylation of pregnane X receptor protein determines selective function independent of ligand activation. Biochem Biophys Res Commun 406:371-376.

Blair SA, Kane SV, Clayburgh DR, and Turner JR (2006) Epithelial myosin light chain kinase expression and activity are upregulated in inflammatory bowel disease. Lab Invest 86:191-201.

Blumberg B, Sabbagh W, Jr, Juguilon H, Bolado J, Jr, van Meter CM, Ong ES, and Evans RM (1998) SXR, a novel steroid and xenobiotic-sensing nuclear receptor. Genes Dev 12:3195-3205.

Bourguet W, Germain P, and Gronemeyer H (2000) Nuclear receptor ligand-binding domains: three-dimensional structures, molecular interactions and pharmacological implications. Trends Pharmacol Sci 21:381-388.

Brown GR, Lindberg G, Meddings J, Silva M, Beutler B, and Thiele D (1999) Tumor necrosis factor inhibitor ameliorates murine intestinal graft-versus-host disease. Gastroenterology 116:593-601.

Carnahan VE and Redinbo MR (2005) Structure and function of the human nuclear xenobiotic receptor PXR. Curr Drug Metab 6:357-367.

Carwile JL and Michels KB (2011) Urinary bisphenol A and obesity: NHANES 20032006. Environ Res 111:825-830.

Chai X, Zeng S, and Xie W (2013) Nuclear receptors PXR and CAR: implications for drug metabolism regulation, pharmacogenomics and beyond. Expert Opin Drug Metab Toxicol 9:253-266.

Chen Y and Nie D (2009) Pregnane X receptor and its potential role in drug resistance in cancer treatment. Recent Patents Anticancer Drug Discov 4:19-27.

Chesik D, De Keyser J, Bron R, and Fuhler GM (2010) Insulin-like growth factor binding protein-1 activates integrin-mediated intracellular signaling and migration in oligodendrocytes. J Neurochem 113:1319-1330.

Christians U, Schmitz V, and Haschke M (2005) Functional interactions between P-glycoprotein and CYP3A in drug metabolism. Expert Opin Drug Metab Toxicol 1 641-654

Cui JY, Gunewardena SS, Rockwell CE, and Klaassen CD (2010) ChIPing the cistrome of PXR in mouse liver. Nucleic Acids Res 38:7943-7963.

Ding X and Staudinger JL (2005a) Induction of drug metabolism by forskolin: the role of the pregnane $\mathrm{X}$ receptor and the protein kinase a signal transduction pathway. $J$ Pharmacol Exp Ther 312:849-856.

Ding X and Staudinger JL (2005b) Repression of PXR-mediated induction of hepatic CYP3A gene expression by protein kinase C. Biochem Pharmacol 69:867-873.

Dring MM, Goulding CA, Trimble VI, Keegan D, Ryan AW, Brophy KM, Smyth CM Keeling PW, O'Donoghue D, O'Sullivan M, et al. (2006) The pregnane X receptor locus is associated with susceptibility to inflammatory bowel disease. Gastroenterology 130:341-348, quiz 592.

Dubrac S, Elentner A, Ebner S, Horejs-Hoeck J, and Schmuth M (2010) Modulation of T lymphocyte function by the pregnane X receptor. J Immunol 184:2949-2957.

Duerksen DR, Wilhelm-Boyles C, and Parry DM (2005) Intestinal permeability in long-term follow-up of patients with celiac disease on a gluten-free diet. Dig Dis Sci 50:785-790.

Eijkelenboom A and Burgering BM (2013) FOXOs: signalling integrators for homeostasis maintenance. Nat Rev Mol Cell Biol 14:83-97.

Elias A, Wu J, and Chen T (2013) Tumor suppressor protein p53 negatively regulates human pregnane X receptor activity. Mol Pharmacol 83:1229-1236.
Fadeel B and Orrenius S (2005) Apoptosis: a basic biological phenomenon with wideranging implications in human disease. J Intern Med 258:479-517.

Febbraio M, Podrez EA, Smith JD, Hajjar DP, Hazen SL, Hoff HF, Sharma K, and Silverstein RL (2000) Targeted disruption of the class B scavenger receptor CD36 protects against atherosclerotic lesion development in mice. J Clin Invest 105:1049-1056.

Forman BM, Tzameli I, Choi HS, Chen J, Simha D, Seol W, Evans RM, and Moore DD (1998) Androstane metabolites bind to and deactivate the nuclear receptor CAR-beta. Nature 395:612-615.

Glass CK and Rosenfeld MG (2000) The coregulator exchange in transcriptional functions of nuclear receptors. Genes Dev 14:121-141.

Goodwin B, Hodgson E, and Liddle C (1999) The orphan human pregnane X receptor mediates the transcriptional activation of CYP3A4 by rifampicin through a distal enhancer module. Mol Pharmacol 56:1329-1339.

Gronemeyer H, Gustafsson JA, and Laudet V (2004) Principles for modulation of the nuclear receptor superfamily. Nat Rev Drug Discov 3:950-964.

Guy E, Kuchibhotla S, Silverstein R, and Febbraio M (2007) Continued inhibition of atherosclerotic lesion development in long term Western diet fed CD36o /apoEo mice. Atherosclerosis 192:123-130.

Hakem R (2008) DNA-damage repair; the good, the bad, and the ugly. EMBO $J$ 27: $589-605$.

Handschin C and Meyer UA (2003) Induction of drug metabolism: the role of nuclear receptors. Pharmacol Rev 55:649-673.

Hariparsad N, Chu X, Yabut J, Labhart P, Hartley DP, Dai X, and Evers R (2009) Identification of pregnane-X receptor target genes and coactivator and corepressor binding to promoter elements in human hepatocytes. Nucleic Acids Res 37: $1160-1173$

Harris RZ, Jang GR, and Tsunoda S (2003) Dietary effects on drug metabolism and transport. Clin Pharmacokinet 42:1071-1088.

Heller F, Florian P, Bojarski C, Richter J, Christ M, Hillenbrand B, Mankertz J, Gitter AH, Bürgel N, Fromm M, et al. (2005) Interleukin-13 is the key effector Th2 cytokine in ulcerative colitis that affects epithelial tight junctions, apoptosis, and cell restitution. Gastroenterology 129:550-564.

Houtgraaf JH, Versmissen J, and van der Giessen WJ (2006) A concise review of DNA damage checkpoints and repair in mammalian cells. Cardiovasc Revasc Med 7: $165-172$.

Huynh H (2004) Overexpression of tumour suppressor retinoblastoma 2 protein (pRb2/p130) in hepatocellular carcinoma. Carcinogenesis 25:1485-1494.

Jones JI and Clemmons DR (1995) Insulin-like growth factors and their binding proteins: biological actions. Endocr Rev 16:3-34.

Kawana K, Ikuta T, Kobayashi Y, Gotoh O, Takeda K, and Kawajiri K (2003) Molecular mechanism of nuclear translocation of an orphan nuclear receptor, SXR. Mol Pharmacol 63:524-531.

Kerr JF, Wyllie AH, and Currie AR (1972) Apoptosis: a basic biological phenomenon with wide-ranging implications in tissue kinetics. Br J Cancer 26:239-257.

Kliewer SA, Moore JT, Wade L, Staudinger JL, Watson MA, Jones SA, McKee DD, Oliver BB, Willson TM, Zetterström RH, et al. (1998) An orphan nuclear receptor activated by pregnanes defines a novel steroid signaling pathway. Cell 92:73-82.

Kodama S and Negishi M (2011) Pregnane X receptor PXR activates the GADD45beta gene, eliciting the p38 MAPK signal and cell migration. J Biol Chem 286: $3570-3578$

Kodama S, Yamazaki Y, and Negishi M (2015) Pregnane X receptor represses HNF4 $\alpha$ gene to induce insulin-like growth factor-binding protein IGFBP1 that alters morphology of and migrates HepG2 cells. Mol Pharmacol 88:746-757.

Konno Y, Negishi M, and Kodama S (2008) The roles of nuclear receptors CAR and PXR in hepatic energy metabolism. Drug Metab Pharmacokinet 23:8-13.

Koyano S, Kurose K, Saito Y, Ozawa S, Hasegawa R, Komamura K, Ueno K, Kamakura S, Kitakaze M, Nakajima T, et al. (2004) Functional characterization of four naturally occurring variants of human pregnane X receptor (PXR): one variant causes dramatic loss of both DNA binding activity and the transactivation of the CYP3A4 promoter/enhancer region. Drug Metab Dispos 32:149-154.

Langmann T, Moehle C, Mauerer R, Scharl M, Liebisch G, Zahn A, Stremmel W, and Schmitz G (2004) Loss of detoxification in inflammatory bowel disease: dysregulation of pregnane X receptor target genes. Gastroenterology 127:26-40.

Lichti-Kaiser K, Xu C, and Staudinger JL (2009) Cyclic AMP-dependent protein kinase signaling modulates pregnane $\mathrm{x}$ receptor activity in a species-specific manner. $J$ Biol Chem 284:6639-6649.

Liebermann DA and Hoffman B (2008) Gadd45 in stress signaling. J Mol Signal 3:15. Lin W, Wu J, Dong H, Bouck D, Zeng FY, and Chen T (2008) Cyclin-dependent kinase 2 negatively regulates human pregnane X receptor-mediated CYP3A4 gene expression in HepG2 liver carcinoma cells. J Biol Chem 283:30650-30657.

Mangelsdorf DJ, Thummel C, Beato M, Herrlich P, Schütz G, Umesono K, Blumberg B, Kastner P, Mark M, Chambon P, et al. (1995) The nuclear receptor superfamily: the second decade. Cell 83:835-839.

Masuyama H, Hiramatsu Y, Kunitomi M, Kudo T, and MacDonald PN (2000) Endocrine disrupting chemicals, phthalic acid and nonylphenol, activate pregnane $\mathrm{X}$ receptor-mediated transcription. Mol Endocrinol 14:421-428.

Masuyama H, Inoshita H, Hiramatsu Y, and Kudo T (2002) Ligands have various potential effects on the degradation of pregnane $\mathrm{X}$ receptor by proteasome. Endocrinology 143:55-61.

Masuyama H, Nakatsukasa H, Takamoto N, and Hiramatsu Y (2007) Down-regulation of pregnane $\mathrm{X}$ receptor contributes to cell growth inhibition and apoptosis by anticancer agents in endometrial cancer cells. Mol Pharmacol 72:1045-1053.

McKenna NJ, Lanz RB, and O'Malley BW (1999) Nuclear receptor coregulators: cellular and molecular biology. Endocr Rev 20:321-344.

Misawa A, Inoue J, Sugino Y, Hosoi H, Sugimoto T, Hosoda F, Ohki M, Imoto I, and Inazawa J (2005) Methylation-associated silencing of the nuclear receptor 1I2 gene in advanced-type neuroblastomas, identified by bacterial artificial chromosome array-based methylated $\mathrm{CpG}$ island amplification. Cancer Res 65 10233-10242. 
Naspinski C, Gu X, Zhou GD, Mertens-Talcott SU, Donnelly KC, and Tian Y (2008) Pregnane X receptor protects HepG2 cells from BaP-induced DNA damage. Toxicol Sci 104:67-73.

Newbold RR, Padilla-Banks E, Snyder RJ, Phillips TM, and Jefferson WN (2007) Developmental exposure to endocrine disruptors and the obesity epidemic. Reprod Toxicol 23:290-296.

Niu Y, Wang Z, Huang H, Zhong S, Cai W, Xie Y, and Shi G (2014) Activated pregnane $\mathrm{X}$ receptor inhibits cervical cancer cell proliferation and tumorigenicity by inducing G2/M cell-cycle arrest. Cancer Lett 347:88-97.

Noble SM, Carnahan VE, Moore LB, Luntz T, Wang H, Ittoop OR, Stimmel JB, Davis-Searles PR, Watkins RE, Wisely GB, et al. (2006) Human PXR forms a tryptophan zipper-mediated homodimer. Biochemistry 45:8579-8589.

Nowack R, Andrassy J, Fischereder M, and Unger M (2009) Effects of dietary factors on drug transport and metabolism: the impact on dosage guidelines in transplant patients. Clin Pharmacol Ther 85:439-443.

Orans J, Teotico DG, and Redinbo MR (2005) The nuclear xenobiotic receptor pregnane X receptor: recent insights and new challenges. Mol Endocrinol 19: $2891-2900$

Ouyang N, Ke S, Eagleton N, Xie Y, Chen G, Laffins B, Yao H, Zhou B, and Tian Y (2010) Pregnane $X$ receptor suppresses proliferation and tumourigenicity of colon cancer cells. Br J Cancer 102:1753-1761.

Poon PY, Kwok HH, Yue PYK, Yang MS, Mak NK, Wong CK, and Wong RN (2012) Cytoprotective effect of 20S-Rg3 on benzo[a]pyrene-induced DNA damage. Drug Metab Dispos 40:120-129.

Qiao E, Ji M, Wu J, Ma R, Zhang X, He Y, Zha Q, Song X, Zhu LW, and Tang J (2013) Expression of the PXR gene in various types of cancer and drug resistance. Oncol Lett 5:1093-1100.

Rana R, Coulter S, Kinyamu H, and Goldstein JA (2013) RBCK1, an E3 ubiquitin ligase, interacts with and ubiquinates the human pregnane X receptor. Drug Metab Dispos 41:398-405.

Robbins D, Cherian M, Wu J, and Chen T (2016) Human pregnane X receptor compromises the function of p53 and promotes malignant transformation. Cell Death Dis 2:16023.

Rosenfeld JM, Vargas R, Jr, Xie W, and Evans RM (2003) Genetic profiling defines the xenobiotic gene network controlled by the nuclear receptor pregnane $\mathrm{X}$ receptor. Mol Endocrinol 17:1268-1282.

Salvador JM, Brown-Clay JD, and Fornace AJ, Jr (2013) Gadd45 in stress signaling, cell cycle control, and apoptosis. Adv Exp Med Biol 793:1-19.

Shizu R, Benoki S, Numakura Y, Kodama S, Miyata M, Yamazoe Y, and Yoshinari K (2013) Xenobiotic-induced hepatocyte proliferation associated with constitutive active/androstane receptor (CAR) or peroxisome proliferator-activated receptor a $(\mathrm{PPAR} \alpha)$ is enhanced by pregnane $\mathrm{X}$ receptor (PXR) activation in mice. PLoS One 8:e61802.

Shizu R, Abe T, Benoki S, Takahashi M, Kodama S, Miayata M, Matsuzawa A and Yoshinari K (2016) PXR stimulates growth factor-mediated hepatocyte proliferation by cross-talk with the FOXO transcription factor. Biochem $J$ 473: $257-266$

Spruiell K, Jones DZ, Cullen JM, Awumey EM, Gonzalez FJ, and Gyamfi MA (2014a) Role of human pregnane $\mathrm{X}$ receptor in high fat diet-induced obesity in premenopausal female mice. Biochem Pharmacol 89:399-412.

Spruiell K, Richardson RM, Cullen JM, Awumey EM, Gonzalez FJ, and Gyamfi MA (2014b) Role of pregnane X receptor in obesity and glucose homeostasis in male mice. J Biol Chem 289:3244-3261.

Squires EJ, Sueyoshi T, and Negishi M (2004) Cytoplasmic localization of pregnane X receptor and ligand-dependent nuclear translocation in mouse liver. $J$ Biol Chem 279:49307-49314.

Suenaert P, Bulteel V, Lemmens L, Noman M, Geypens B, Van Assche G, Geboes K, Ceuppens JL, and Rutgeerts P (2002) Anti-tumor necrosis factor treatment restores the gut barrier in Crohn's disease. Am J Gastroenterol 97:2000-2004

Sugatani J, Osabe M, Kurosawa M, Kitamura N, Ikari A, and Miwa M (2010) Induction of UGT1A1 and CYP2B6 by an antimitogenic factor in HepG2 cells is mediated through suppression of cyclin-dependent kinase 2 activity: cell cycledependent expression. Drug Metab Dispos 38:177-186.

Sugatani J, Uchida T, Kurosawa M, Yamaguchi M, Yamazaki Y, Ikari A, and Miwa M (2012) Regulation of pregnane X receptor (PXR) function and UGT1A1 gene expression by posttranslational modification of PXR protein. Drug Metab Dispos 40 2031-2040.

Sun D, Ren H, Oertel M, Sellers RS, Shafritz DA, and Zhu L (2008) Inactivation of p27Kip1 promotes chemical mouse liver tumorigenesis in the resistant strain C57BL/6J. Mol Carcinog 47:47-55.

Takeshita A, Koibuchi N, Oka J, Taguchi M, Shishiba Y, and Ozawa Y (2001) Bisphenol-A, an environmental estrogen, activates the human orphan nuclear receptor, steroid and xenobiotic receptor-mediated transcription. Eur $J$ Endocrinol 145:513-517.

Tamion F, Richard V, Lyoumi S, Daveau M, Bonmarchand G, Leroy J, Thuillez C, and Lebreton JP (1997) Gut ischemia and mesenteric synthesis of inflammatory cytokines after hemorrhagic or endotoxic shock. Am J Physiol 273:G314-G321.

Thatcher NJ and Caldwell J (1994) Origins of hepatomegaly produced by dexamethasone (DEX), pregnenolone 16 alpha-carbonitrile (PCN) and phenobarbitone (PB) in female Sprague-Dawley rats. Biochem Soc Trans 22:132S.

Timsit YE and Negishi M (2007) CAR and PXR: the xenobiotic-sensing receptors. Steroids 72:231-246.

Turner JR (2009) Intestinal mucosal barrier function in health and disease. Nat Rev Immunol 9:799-809.
Umesono K and Evans RM (1989) Determinants of target gene specificity for steroid/ thyroid hormone receptors. Cell 57:1139-1146.

van de Winkel A, Menke V, Capello A, Moons LM, Pot RG, van Dekken H, Siersema PD, Kusters JG, van der Laan LJ, and Kuipers EJ (2011) Expression, localization and polymorphisms of the nuclear receptor PXR in Barrett's esophagus and esophageal adenocarcinoma. BMC Gastroenterol 11:108.

Venkatesh M, Mukherjee S, Wang H, Li H, Sun K, Benechet AP, Qiu Z, Maher L, Redinbo MR, Phillips RS, et al. (2014) Symbiotic bacterial metabolites regulate gastrointestinal barrier function via the xenobiotic sensor PXR and toll-like receptor 4. Immunity 41:296-310.

vom Saal FS and Myers JP (2008) Bisphenol A and risk of metabolic disorders. JAMA 300:1353-1355.

Wada T, Gao J, and Xie W (2009) PXR and CAR in energy metabolism. Trends Endocrinol Metab 20:273-279.

Wallace BD, Betts L, Talmage G, Pollet RM, Holman NS, and Redinbo MR (2013) Structural and functional analysis of the human nuclear xenobiotic receptor PXR in complex with RXR $\alpha$. J Mol Biol 425:2561-2577.

Wang H, Venkatesh M, Li H, Goetz R, Mukherjee S, Biswas A, Zhu L, Kaubisch A, Wang L, Pullman J, et al. (2011) Pregnane X receptor activation induces FGF19dependent tumor aggressiveness in humans and mice. $J$ Clin Invest 121: $3220-3232$

Wang YM, Ong SS, Chai SC, and Chen T (2012) Role of CAR and PXR in xenobiotic sensing and metabolism. Expert Opin Drug Metab Toxicol 8:803-817.

Watkins RE, Wisely GB, Moore LB, Collins JL, Lambert MH, Williams SP, Willson TM, Kliewer SA, and Redinbo MR (2001) The human nuclear xenobiotic receptor PXR: structural determinants of directed promiscuity. Science 292:2329-2333.

Watkins RE, Davis-Searles PR, Lambert MH, and Redinbo MR (2003) Coactivator binding promotes the specific interaction between ligand and the pregnane $\mathrm{X}$ receptor. $J$ Mol Biol 331:815-828.

Wei P, Zhang J, Dowhan DH, Han Y, and Moore DD (2002) Specific and overlapping functions of the nuclear hormone receptors CAR and PXR in xenobiotic response. Pharmacogenomics J 2:117-126.

Wei P, Zhang J, Egan-Hafley M, Liang S, and Moore DD (2000) The nuclear receptor CAR mediates specific xenobiotic induction of drug metabolism. Nature 407: 920-923.

Wei Y, Tang C, Sant V, Li S, Poloyac SM, and Xie W (2016) A molecular aspect in the regulation of drug metabolism: does PXR-induced enzyme expression always lead to functional changes in drug metabolism? Curr Pharmacol Rep 2: $187-192$

Weikum ER, Knuesel MT, Ortlund EA, and Yamamoto KR (2017) Glucocorticoid receptor control of transcription: precision and plasticity via allostery. Nat Rev Mol Cell Biol 18:159-174.

Wikoff WR, Anfora AT, Liu J, Schultz PG, Lesley SA, Peters EC, and Siuzdak G (2009) Metabolomics analysis reveals large effects of gut microflora on mammalian blood metabolites. Proc Natl Acad Sci USA 106:3698-3703.

Xiao L, Zhang Z, and Luo X (2014) Roles of xenobiotic receptors in vascular pathophysiology. Circ J 78:1520-1530.

Xie F, Ding X, and Zhang QY (2016) An update on the role of intestinal cytochrome P450 enzymes in drug disposition. Acta Pharm Sin B 6:374-383.

Xie W and Evans RM (2001) Orphan nuclear receptors: the exotics of xenobiotics. $J$ Biol Chem 276:37739-37742.

Xu C, Huang M, and Bi H (2016) PXR- and CAR-mediated herbal effect on human diseases. Biochim Biophys Acta 1859:1121-1129.

Yan J and Xie W (2016) A brief history of the discovery of PXR and CAR as xenobiotic receptors. Acta Pharm Sin B 6:450-452.

Zeissig S, Bürgel N, Günzel D, Richter J, Mankertz J, Wahnschaffe U, Kroesen AJ, Zeitz M, Fromm M, and Schulzke JD (2007) Changes in expression and distribution of claudin 2,5 and 8 lead to discontinuous tight junctions and barrier dysfunction in active Crohn's disease. Gut 56:61-72.

Zhou C, Tabb MM, Nelson EL, Grün F, Verma S, Sadatrafiei A, Lin M, Mallick S, Forman BM, Thummel KE, et al. (2006a) Mutual repression between steroid and xenobiotic receptor and NF-kappaB signaling pathways links xenobiotic metabolism and inflammation. J Clin Invest 116:2280-2289.

Zhou C, Verma S, and Blumberg B (2009) The steroid and xenobiotic receptor (SXR), beyond xenobiotic metabolism. Nucl Recept Signal 7:e001.

Zhou J, Zhai Y, Mu Y, Gong H, Uppal H, Toma D, Ren S, Evans RM, and Xie W (2006b) A novel pregnane $\mathrm{X}$ receptor-mediated and sterol regulatory elementbinding protein-independent lipogenic pathway. J Biol Chem 281:15013-15020.

Zhou J, Liu M, Zhai Y, and Xie W (2008) The antiapoptotic role of pregnane X receptor in human colon cancer cells. Mol Endocrinol 22:868-880.

Zhuang W, Jia Z, Feng H, Chen J, Wang H, Guo Y, and Meng C (2011) The mechanism of the G0/G1 cell cycle phase arrest induced by activation of PXR in human cells. Biomed Pharmacother 65:467-473.

Zucchini N, de Sousa G, Bailly-Maitre B, Gugenheim J, Bars R, Lemaire G, and Rahmani R (2005) Regulation of Bcl-2 and Bcl-xL anti-apoptotic protein expression by nuclear receptor PXR in primary cultures of human and rat hepatocytes. Biochim Biophys Acta 1745:48-58.

Address correspondence to: Dr. Taosheng Chen, Department of Chemical Biology and Therapeutics, St. Jude Children's Research Hospital, Mail Stop \#1000 262 Danny Thomas Place, Memphis, TN 38105. E-mail: taosheng.chen@ stjude.org 